\title{
Nanosatellite Air Bearing Tests of Fault-Tolerant Sliding-Mode Attitude Control with Unscented Kalman Filter
}

\author{
Mark A. Post* \\ York University, 4700 Keele Street Toronto, Ontario M3J 1P3, Canada \\ Junquan $\mathrm{Li}^{\dagger}$ \\ Ryerson University, 350 Victoria St., Toronto, Ontario, M5B 2K3, Canada \\ Regina Lee ${ }^{\ddagger}$ \\ York University, 4700 Keele Street Toronto, Ontario M3J 1P3, Canada
}

\begin{abstract}
This paper documents the development and testing of a fault tolerant sliding mode attitude control algorithm for a nanosatellite with reaction wheel control actuation. A locally asymptotically stable adaptive fuzzy first order sliding mode controller is used to solve the local attitude control tracking problem. An adaptive unscented Kalman filter with a reduced sigma set is proposed to improve estimation of nanosatellite attitude on an air bearing test system and and compared in simulation with a symmetric adaptive unscented Kalman filter. Tests include a comparison with PID, second order and third order sliding mode controllers, and the results validate the performance of the proposed adaptive fuzzy sliding mode controller and unscented Kalman filter on embedded nanosatellite hardware in the presence of noise and faults.
\end{abstract}

\section{Introduction}

Air bearings have been used for satellite attitude determination, control hardware verification, and software system testing for nearly 54 years. ${ }^{1}$ Spherical air bearings that use a cushion of compressed air to provide nearly friction-free rotation about any axis are one of the most common devices used for ADCS testing. Such a friction-free spherical air bearing system is used for testing nanosatellite nonlinear attitude control systems at York University (as shown in Figs. 1 and 2). Nanosatellites and other small satellites, which can have a mass of just a few kilograms, typically use lower-cost and lower-accuracy components and have less hardware redundancy available to handle hardware faults than large satellites. However, attitude control of small satellites is still dominated by linear controllers such as Proportional-Integral-Derivative (PID) controllers, as the complexity of nonlinear attitude control systems hinders the development of practical implementations. For precise autonomous attitude control of such a satellite, a robust but compact nonlinear estimation and control algorithm is desirable. In previous work, ${ }^{2}$ a fault tolerant sliding-mode controller (SMC) was developed for a $1 \mathrm{U}$ cubesat. To overcome faults and inaccuracies injected into the hardware, adaptive sliding mode control laws with a fuzzy system (AFSMC) were developed and tested on embedded nanosatellite hardware with reaction wheels for attitude control. The embedded version of the controller was programmed natively in the C language, and a flow chart of the ACS software is shown in Fig. 3. A similar approach with an air bearing system has been used at the Surrey space center. ${ }^{3}$

The state and observation models for a satellite are inherently nonlinear. Satellite attitude determination with nonlinear filters has been studied by many researchers. In the reference, ${ }^{4}$ many nonlinear filtering methods for spacecraft attitude determination have been surveyed. The Unscented Kalman filter (UKF) is one of the most popular nonlinear filters for ACS. ${ }^{5}$ Due to the use of statistical estimation in the UKF, models

*PhD Candidate, Department of Earth and Space Engineering; markpost@yorku.ca.

${ }^{\dagger} \mathrm{PhD}$ Candidate, Department of Aerospace Engineering; junquan.li@ryerson.ca.

¥Assistant Professor, Department of Earth and Space Engineering; reginal@yorku.ca. 
do not require linearization and the Jacobians and Hessians do not need to be calculated as in the Extended Kalman Filter. The prior statistical distributions of the state variables are encapsulated with sigma points (state space samples) and transformed to posterior distributions using a system model, as shown in Figure 4. Making appropriate assumptions of the prior covariances is key to achieving good estimation results, but most UKF implementations assume that the statistics and covariances remain constant. One way to overcome this limitation is to use an adaptive algorithm for the UKF statistics. There are several papers on using adaptive Kalman filters ${ }^{678}$.

The Unscented Kalman filter has been often used for parameter estimation because of its ability to handle nonlinear systems very well. ${ }^{9}$ The formulation for parameter estimation with a UKF includes the computation of weights, the establishment of sigma points, the prediction of the mean and covariance of both states and measurements, the prediction of cross covariance, the gain calculation, and the state update step. However, the computational load is a limiting factor, which is proportional to the number of sigma points. There are three common sets of sigma points used in the literature with $n$ state variables: the symmetric set $\left(2 n+1\right.$ points), the reduced set ( $n+1$ points), and the spherical set $\left(n+2\right.$ points). In a recent publication, ${ }^{10}$ a new minimum sigma set with equal size to the reduced set but better high-order performance is proposed and applied to localization and map building.

The air bearing system uses analog gyroscopic rate sensors for rotational feedback, which experience slow angular drift over time. An Adaptive Unscented Kalman Filter is used to compensate for drift and sensor noise in the system and increase the accuracy of the sliding mode controller. Our main contribution is to apply this new sigma set which uses $n+1$ sigma points to the adaptive unscented Kalman filter in the satellite attitude air bearing system. The benefits of this reduced AUKF are the use of less sigma points and less computational load while achieving good estimation results.

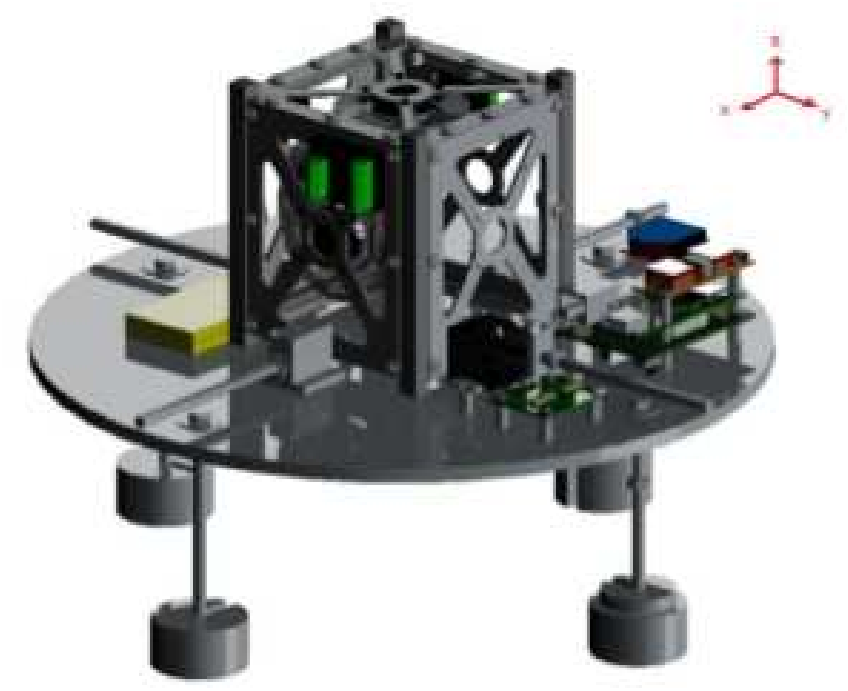

Figure 1. Attitude Control Test Platform Model, York University Nanosatellite Lab

\section{New Adaptive Unscented Kalman Filter}

For highly non-linear satellite attitude control systems with large disturbances, the adaptive unscented Kalman filter (AUKF) is a good choice for the reasons stated above. The formulation for parameter estimation with the AUKF is shown as follows. Consider the discrete nonlinear system:

$$
\begin{gathered}
X_{k+1}=f\left(X_{k}, u_{k}, k\right)+\eta_{s k} \\
Z_{K+1}=h\left(X_{k+1}, u_{k+1}, k+1\right)+\eta_{o k+1}
\end{gathered}
$$

where $X_{k} \in R_{L}$ is the state vector, $Z_{k} \in R_{M}$ is the output vector at time $\mathrm{k}, u_{k}$ is the control input at time $\mathrm{k}, d_{k}$ is the disturbance at time $\mathrm{k}, \eta_{s k}$ is the white noise with a mean of $q_{k}$ and covariance $Q_{k}$, and $\eta_{o k}$ is the white noise with a mean of $r_{k}$ and covariance $R_{k}$. 


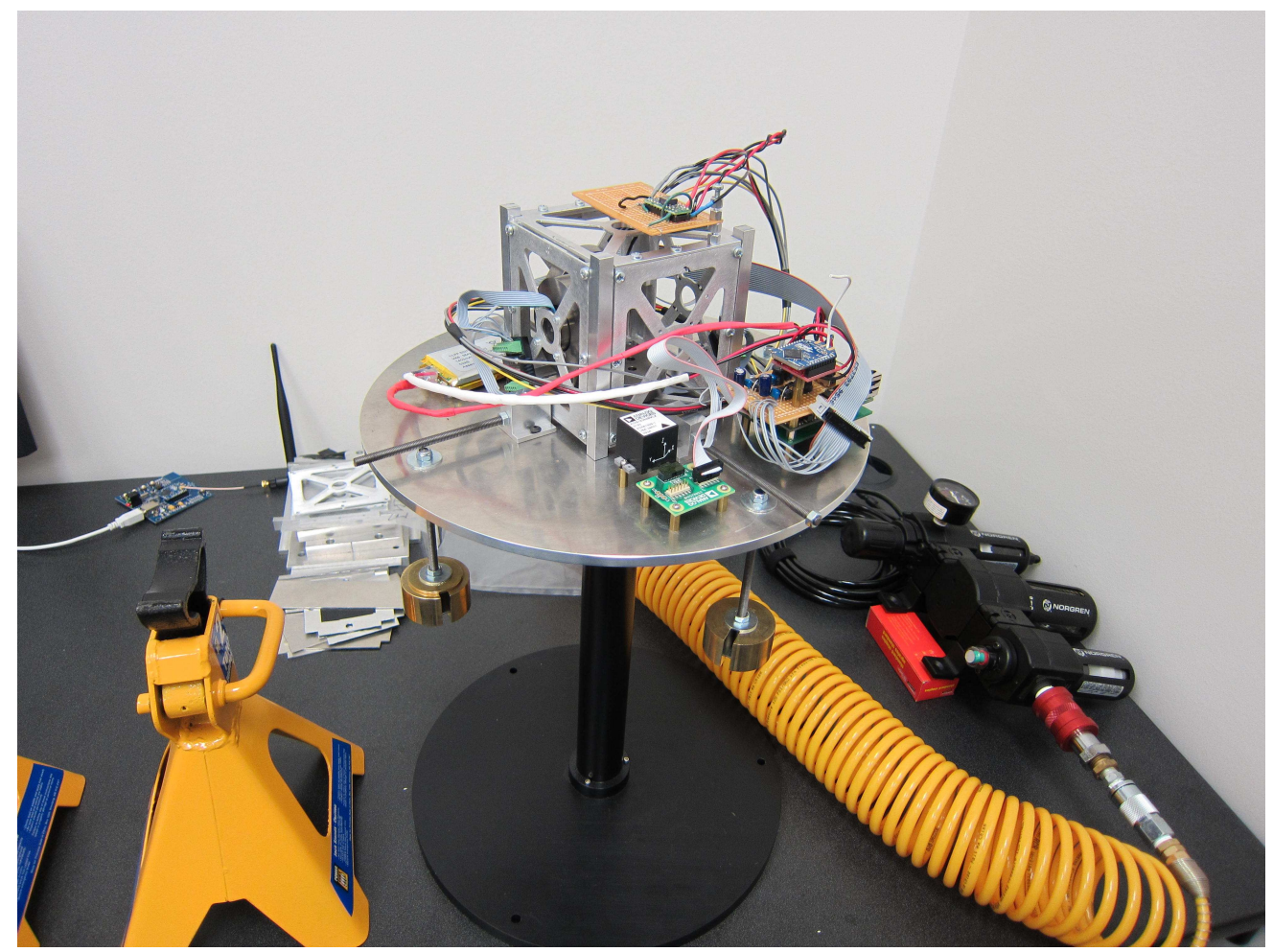

Figure 2. Spherical Air Bearing Setup with Electronics System and Control System

The conventional $\mathrm{UKF}^{11}$ is based on the determination of $2 n+1$ sigma points, commonly known as the symmetric set. The sigma points are obtained by

$$
\begin{gathered}
\hat{\chi}_{0}=\hat{x}_{k+1} \\
\hat{\chi}_{i, k+1}=\hat{x}_{k+1}+\sqrt{(n+\kappa) P_{(k+1) i}}, \quad \forall i=1,2, \ldots, n \\
\hat{\chi}_{i, k+q}=\hat{x}_{k+1}-\sqrt{(n+\kappa) P_{(k+1) i}} \quad \forall i=n+1, \ldots, 2 n
\end{gathered}
$$

where $\hat{\chi}_{i, k}$ are sigma points, $\mathrm{n}$ is the state number and $\kappa$ is the scaling parameter. The next step is prediction. Each point is run through the nonlinear system model to yield a set of transformed points

$$
\hat{\chi}_{i, k \mid k+1}=f\left(\hat{\chi}_{i, k+1}\right)+q_{k+1}
$$

The transformed values are utilized for gaining the predicted mean and covariance. This process, the Unscented Transform, is illustrated in Figure 4.

$$
\begin{gathered}
\hat{x}_{k \mid k+1}=\sum_{i=0}^{2 n} W_{i}^{m} \hat{\chi}_{i, k \mid k+1} \\
P_{k \mid k+1}=\sum_{i=0}^{2 n} W_{i}^{c}\left(\hat{\chi}_{i, k \mid k+1}-\hat{x}_{k \mid k+1}\right)\left(\hat{\chi}_{i, k \mid k+1}-\hat{x}_{k \mid k+1}\right)^{T}+Q_{k+1} \\
W_{i}^{m}=\frac{\kappa}{n+\kappa} \\
W_{i}^{c}=\frac{1}{2(n+\kappa)}
\end{gathered}
$$

The predicted observation vector $\bar{y}_{k \mid k+1}$ and its predicted covariance $P_{y y}$ are defined by

$$
Y_{i, k \mid k+1}=h\left(\hat{\chi}_{i, k \mid k+1}, k+1\right)+r_{k+1}
$$



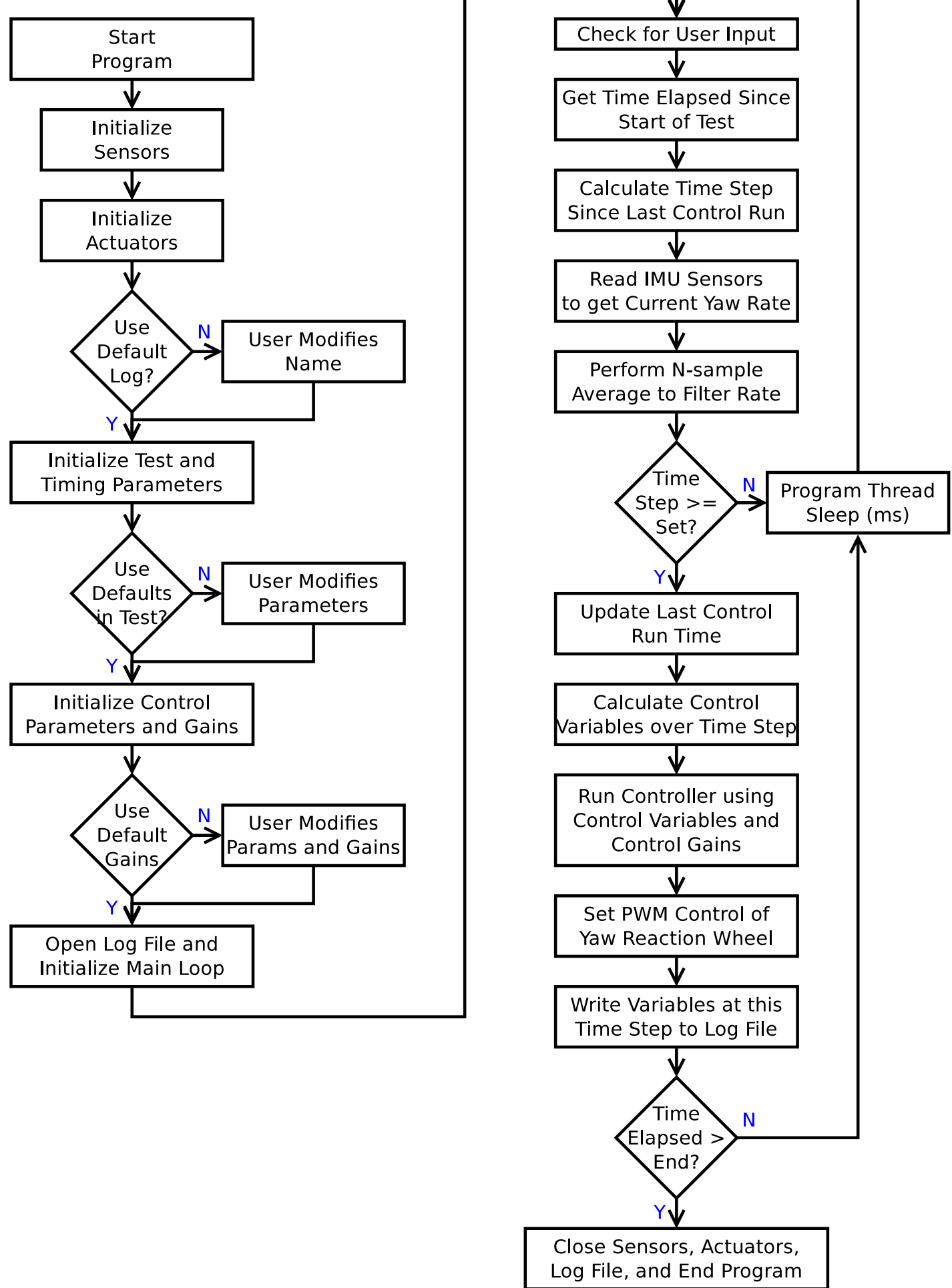

Figure 3. Software Flow Chart of Spherical Air Bearing System 


$$
\begin{gathered}
\bar{y}_{k \mid k+1}=\sum_{i=0}^{2 n} W_{i}^{m} Y_{i, k \mid k+1}+R_{k+1} \\
P_{y y}=\sum_{i=0}^{2 n} W_{i}^{c}\left(Y_{i, k \mid k+1}-\bar{y}_{k \mid k+1}\right)\left(Y_{i, k \mid k+1}-\bar{y}_{k \mid k+1}\right)^{T} \\
P_{x y}=\sum_{i=0}^{2 n} W_{i}^{c}\left(\hat{\chi}_{i, k \mid k+1}-\hat{x}_{k \mid k+1}\right)\left(Y_{i, k \mid k+1}-\bar{y}_{k \mid k+1}\right)^{T} \\
K_{k}=P_{x y} P_{y y}^{-1} \\
\hat{x}_{k}=\hat{x}_{k \mid k+1}+K_{k}\left(Y_{k}-\bar{y}_{k \mid k+1}\right) \\
P_{k}=P_{k \mid k+1}-K_{k} P_{y y} K_{k}^{T}
\end{gathered}
$$

If the prior statistics of the noise are not known or change over time, an adaptive algorithm can be used to adjust the noise covariance matrices $Q$ and $R$ as shown in Figure 5. In this study, the statistical estimator is based on the reference, ${ }^{6}$ which is applied as follows. An estimate of the innovation covariance is obtained by averaging the innovation sequence over a window of length $N$

$$
\hat{C}_{v}=\frac{1}{N} \sum_{j=k-N+1}^{k} \Delta \hat{x}_{k} \Delta \hat{x}_{k}^{T}
$$

Then based on the whiteness of the filter innovation sequence the statistical matrices can be estimated as

$$
\begin{gathered}
\hat{R}_{k}=\hat{C}_{v}+P_{k \mid k+1}-K_{k+1} P_{y y} K_{k+1}^{T} \\
\hat{Q}_{k}=K_{k+1} \hat{C}_{v} K_{k+1}^{T}
\end{gathered}
$$

The unscented Kalman filter's computational load is proportional to the number of sigma points which is related to the number of states. The following new asymmetric minimum sigma sets of size $n+1$ are based on the reference, ${ }^{10}$ and modify the sigma set as shown in Figure 6.

$$
\begin{gathered}
W_{0}=\frac{\kappa}{n+\kappa} \\
\alpha=\sqrt{\frac{1-W_{0}}{n}} \\
C=\sqrt{I_{n}-\alpha^{2} * 1_{n}}
\end{gathered}
$$

where $0<W_{0}<1$.

The new sigma points are obtained by

$$
\begin{gathered}
\hat{\chi}_{0}=\hat{x}_{k+1}-\frac{\sqrt{(n+\kappa) P_{(k+1) i}}}{\sqrt{W}_{0}} *[\alpha]_{n \times 1} \\
\hat{\chi}_{i, k+1}=\hat{x}_{k+1}+\sqrt{(n+\kappa) P_{(k+1) i}} * C * I S W_{i}, \quad \forall i=1,2, \ldots, n
\end{gathered}
$$

where $I S W_{i}$ is the Cholesky decomposition of $\operatorname{diag}\left(W_{0} * \alpha^{2} * C^{-1} * 1_{n} *\left(C^{T}\right)^{-1}\right)$.

The design of this new AUKF is similar to the existing AUKF. Eqs. 8, 12, 13 and 14 have to be rewritten with the new defined weights:

$$
\bar{y}_{k \mid k+1}=\sum_{i=0}^{n} \bar{W}_{i} Y_{i, k \mid k+1}+R_{k+1}
$$




$$
\begin{aligned}
P_{y y} & =\sum_{i=0}^{n} \bar{W}_{i}\left(Y_{i, k \mid k+1}-\bar{y}_{k \mid k+1}\right)\left(Y_{i, k \mid k+1}-\bar{y}_{k \mid k+1}\right)^{T} \\
P_{x y} & =\sum_{i=0}^{n} \bar{W}_{i}\left(\hat{\chi}_{i, k \mid k+1}-\hat{x}_{k \mid k+1}\right)\left(Y_{i, k \mid k+1}-\bar{y}_{k \mid k+1}\right)^{T}
\end{aligned}
$$

The weightings $\bar{W}_{i}$ are calculated as the diagonal values of the matrix equation:

$$
\left[\begin{array}{ccc}
\bar{W} 1 & \cdots & \sqrt{\bar{W}_{1}} \sqrt{\bar{W}_{n}} \\
\cdots & \cdots & \cdots \\
\sqrt{\bar{W}_{1}} \sqrt{\bar{W}_{n}} & \cdots & \bar{W}_{n}
\end{array}\right]=W_{0} * \alpha^{2} * C^{-1} * 1_{n} *\left(C^{T}\right)^{-1}
$$

Previous State $\left(\chi_{i}\right)$

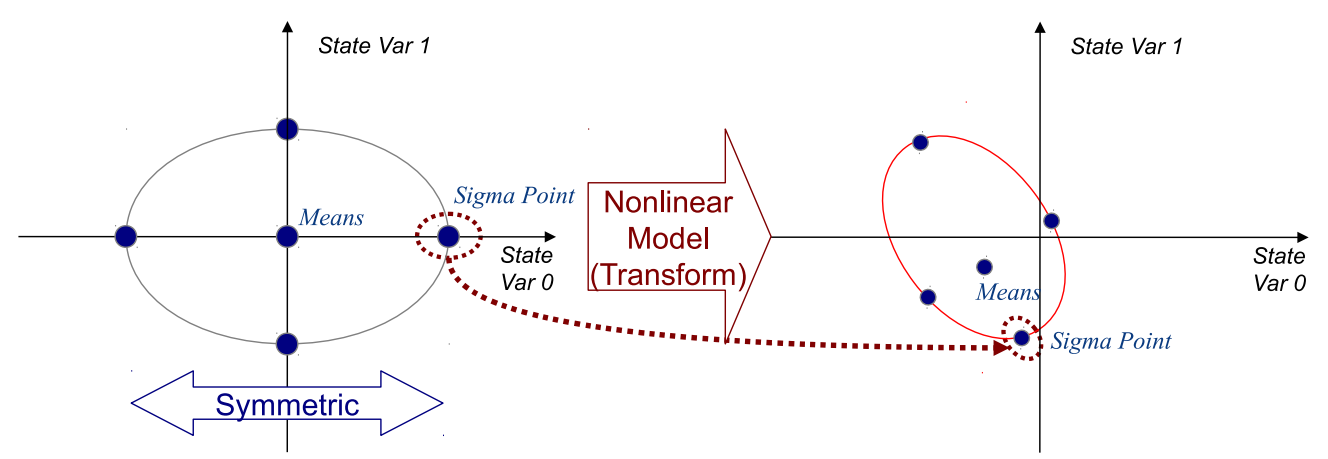

Figure 4. The Unscented Transform, Traditional UKF
Current State $\left(Y_{i}\right)$

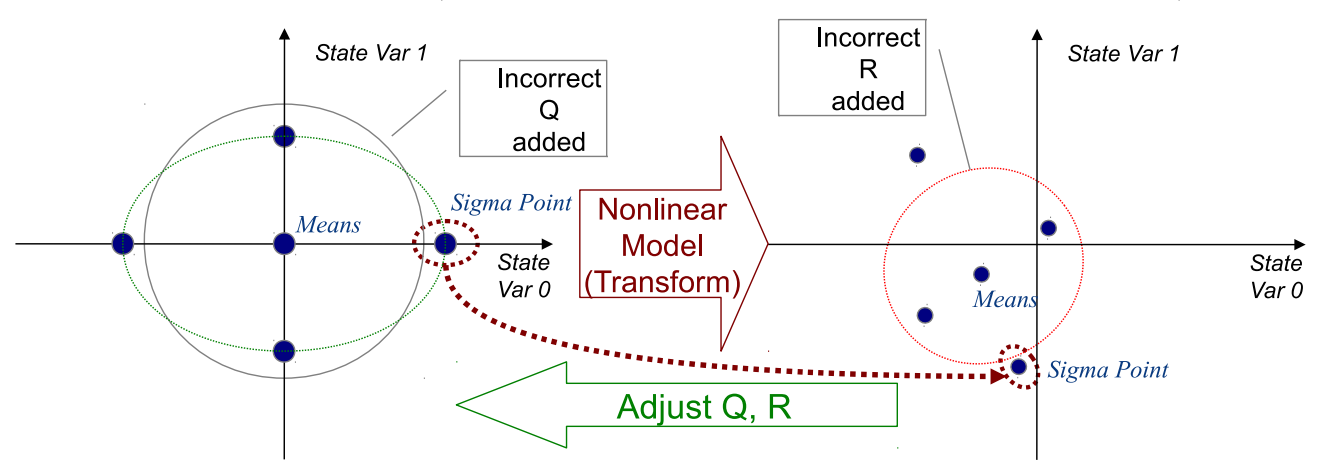

Figure 5. The Unscented Transform, Adaptive UKF

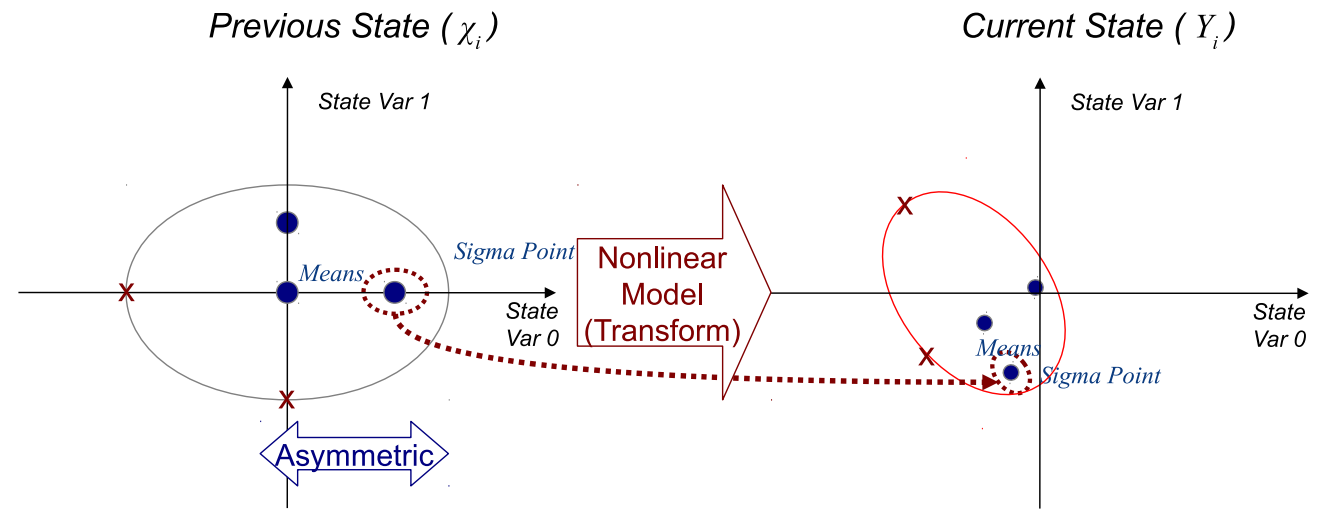

Figure 6. The Unscented Transform, Reduced Sigma Set UKF 


\section{Mathematical Model of the Nanosatellite}

The spacecraft is modelled as a rigid body with reaction wheels to provide torques about three mutually perpendicular axes that define a body-fixed frame $B$. The equations of motion are given by

$$
\begin{aligned}
\hat{J} \dot{\omega} & =-\omega^{\times}\left(J_{s} \omega+A_{i} J_{w} \Omega\right)+A_{i} \tau+d \\
\dot{q} & =\frac{1}{2}\left(\begin{array}{c}
q_{4} I_{3 \times 3}+\bar{q}^{\times} \\
-\bar{q}^{T}
\end{array}\right) \omega \equiv \frac{1}{2} A(q) \omega
\end{aligned}
$$

where $\omega=\left(\omega_{1}, \omega_{2}, \omega_{3}\right)^{T}$ is the angular velocity of the spacecraft with respect to an inertial frame $I$ and expressed in the body frame $B, \Omega$ is the angular velocity of the reaction wheel, $J_{s} \in R^{3 \times 3}$ is the inertia matrix of the spacecraft, $\hat{J}=J_{s}-A_{i} J_{w} A_{i}^{T} ; \tau \in R^{3}$ is the torque control, $A_{i}$ is the $3 \times 4$ or $3 \times 3$ (depending on the layout and the number of reaction wheels) matrix whose columns represent the influence of each reaction wheel on the angular acceleration of the satellite, $d \in R^{3}$ is the bounded external disturbance, $x^{\times} \in R^{3 \times 3}$ represents the cross product operator for a vector $x=\left(x_{1}, x_{2}, x_{3}\right)^{T}$ given as

$$
x^{\times}=\left(\begin{array}{ccc}
0 & -x_{3} & x_{2} \\
x_{3} & 0 & -x_{1} \\
-x_{2} & x_{1} & 0
\end{array}\right)
$$

and the unit quaternion $q=\left(\bar{q}^{T}, q_{4}\right)^{T}=\left(q_{1}, q_{2}, q_{3}, q_{4}\right)^{T}$ represents the attitude orientation of a rigid spacecraft in the body frame $B$ with respect to the inertial frame $I$, which is defined by

$$
\bar{q}=\left(q_{1}, q_{2}, q_{3}\right)^{T}=e \sin (\theta / 2), \quad q_{4}=\cos (\theta / 2)
$$

where $e$ is the Euler axis, and $\theta$ is the Euler angle. The unit quaternion $q$ satisfies the constraint

$$
q^{T} q=1
$$

The torques generated by the reaction wheels $\tau$ are given by

$$
\tau=J_{w}\left(\dot{\Omega}+A_{i}^{T} \dot{\omega}\right)
$$

The angular velocity is measured from the gyro. A well-known model for the angular velocity measurement is given by

$$
\omega_{g}=\omega+b_{g}+\eta_{g}
$$

where $\eta_{g} \in R^{3}$ is the output of gyro, and $\omega$ is the real angular rate of the gyro, $\eta_{g}$ and $\eta_{f}$ are the independent Gaussian white noise with zero mean and standard deviation. $b_{g}$ is the random drift. $k_{f}$ is a constant number.

$$
\dot{b}_{g}=-k_{f} * b_{g}+\eta_{f}
$$

\section{AFSMC Controller}

The nonlinear AFSMC controller used for a satellite in these tests is given by ${ }^{12}$

$$
\begin{aligned}
\tau_{A F S M C} & =-k_{1} s-\theta^{T} \xi-\kappa \tanh \left(\frac{3 K_{u} \kappa s}{\epsilon}\right) \\
\dot{\theta} & =\alpha s \xi
\end{aligned}
$$

where $s$ is the sliding surface. $\alpha, \kappa, \epsilon$ are positive constant numbers. $\xi$ is built using fuzzy membership functions. $H_{r}=\theta^{T} \xi$ is the estimation of the nonlinear dynamics function by a fuzzy logic system.

The PID controller equation used for comparison is

$$
\tau_{P I D}=k_{p} e+k_{i} \int_{0}^{t} e+k_{d} \dot{e}
$$


Additionally, second and third order SMC satellite attitude controllers ${ }^{13}$ are given by

$$
\begin{gathered}
\hat{\tau}_{2 n d S M C}=-k \frac{\dot{s}+|s|^{1 / 2} \operatorname{sign}(s)}{\dot{s}+|s|^{1 / 2}} \\
\bar{\tau}_{3 r d S M C}=-\hat{k} \frac{\ddot{s}+2\left(|\dot{s}|+|s|^{2 / 3}\right)^{-1 / 2}\left(\dot{s}+|s|^{2 / 3} \text { signs }\right)}{|\ddot{s}|+2\left(|\dot{s}|+|s|^{2 / 3}\right)^{1 / 2}}
\end{gathered}
$$

It is difficult to find $\dot{s}$ and $\ddot{s}$. For Eq. 41, a first-order real-time differentiator ${ }^{14} Q_{0}$ and $Q_{1}$ is used in to estimate $s$ and $\dot{s}$.

$$
\begin{aligned}
\dot{Q}_{0} & =-\lambda_{1}\left|Q_{0}-s\right|^{1 / 2} \operatorname{sign}\left(Q_{0}-s\right)+Q_{1} \\
\dot{Q}_{1} & =-\lambda_{2} \operatorname{sign}\left(Q_{0}-s\right) \\
\varphi & \equiv \kappa \tanh \left(\frac{3 K_{u} \kappa Q_{0}}{\epsilon}\right)
\end{aligned}
$$

For Eq. 42, a second-order real-time differentiator ${ }^{14} Q_{0}, Q_{1}$ and $Q_{2}$ is used in to estimate $s, \dot{s}$ and $\ddot{s}$.

$$
\begin{aligned}
\dot{Q}_{0} & =Z_{0} \\
Z_{0} & =-\hat{\lambda}_{1}\left|Q_{0}-s\right|^{2 / 3} \operatorname{sign}\left(Q_{0}-s\right)+Q_{1} \\
\dot{Q}_{1} & =Z_{1} \\
Z_{1} & =-\hat{\lambda}_{2}\left|Q_{1}-Z_{0}\right|^{1 / 2} \operatorname{sign}\left(Q_{1}-Z_{0}\right)+Q_{2} \\
\dot{Q}_{2} & =-\hat{\lambda}_{3} \operatorname{sign}\left(Q_{2}-Z_{1}\right) \\
\varphi & \equiv \kappa \tanh \left(\frac{3 K_{u} \kappa Q_{0}}{\epsilon}\right)
\end{aligned}
$$

\section{Results from Simulated AUKF Coupled with Control Algorithm}

A simulation of the system performance while using the UKF to filter rate sensor feedback is implemented under the following conditions: The nanosatellite is assumed to be a three axis body stabilized in a $500 \mathrm{~km}$ circular orbit with 45 degree inclination. The sampling time is $0.1 \mathrm{~s}$. The proposed reduced-set AUKF is studied in comparison with the existing AUKF. Similar results with a nonlinear observer coupled with the control algorithm can be seen in. ${ }^{15}$ In Fig. 7, the angular velocity of the satellite Z-axis is shown as the real value (solid line), the estimated value using the existing AUKF (dashed line), and the estimated value from the reduced-set AUKF (dash dot line). The estimation of the reduced-set AUKF is faster and has better accuracy. Fig. 8 presents the results of gyro drift estimation using new AUKF and existing AUKF. The solid line with $\star$ markers showing the reduced-set AUKF matches well with the real values. The estimation error between the solid line with $\times$ markers showing the existing AUKF and the real value are larger than that of the reduced-set AUKF. It can be seen that the reduced-set AUKF significantly better estimates the gyro drift.

\section{Results from Embedded Hardware on Air Bearing Platform}

For comparison, tests of the filter and controller were performed on the air bearing system using AFSMC, PID, 2nd order SMC, and 3rd order SMC control laws, with a sampling time of 0.01s and faults injected into the system to verify controller robustness. Experimental results showed that the SMC control laws were effective in dealing with hardware faults and achieved better attitude tracking accuracy than a similar PID controller developed in the reference. ${ }^{16}$

First, single-axis control on the spacecraft Z-axis (yaw) was tested with a 90 degree yaw slew maneuver. Figure 9 shows the satellite angle over time using AFSMC, 2nd order SMC, and 3rd order SMC controllers, with no hardware faults. Control response for all three controllers is similar and predictable. Using the same 90 degree yaw slew maneuver, the AFSMC controller was compared to the aforementioned PID controller with the actuator experiencing a constant speed offset of $25 \%$ of maximum is added to the yaw wheel between 


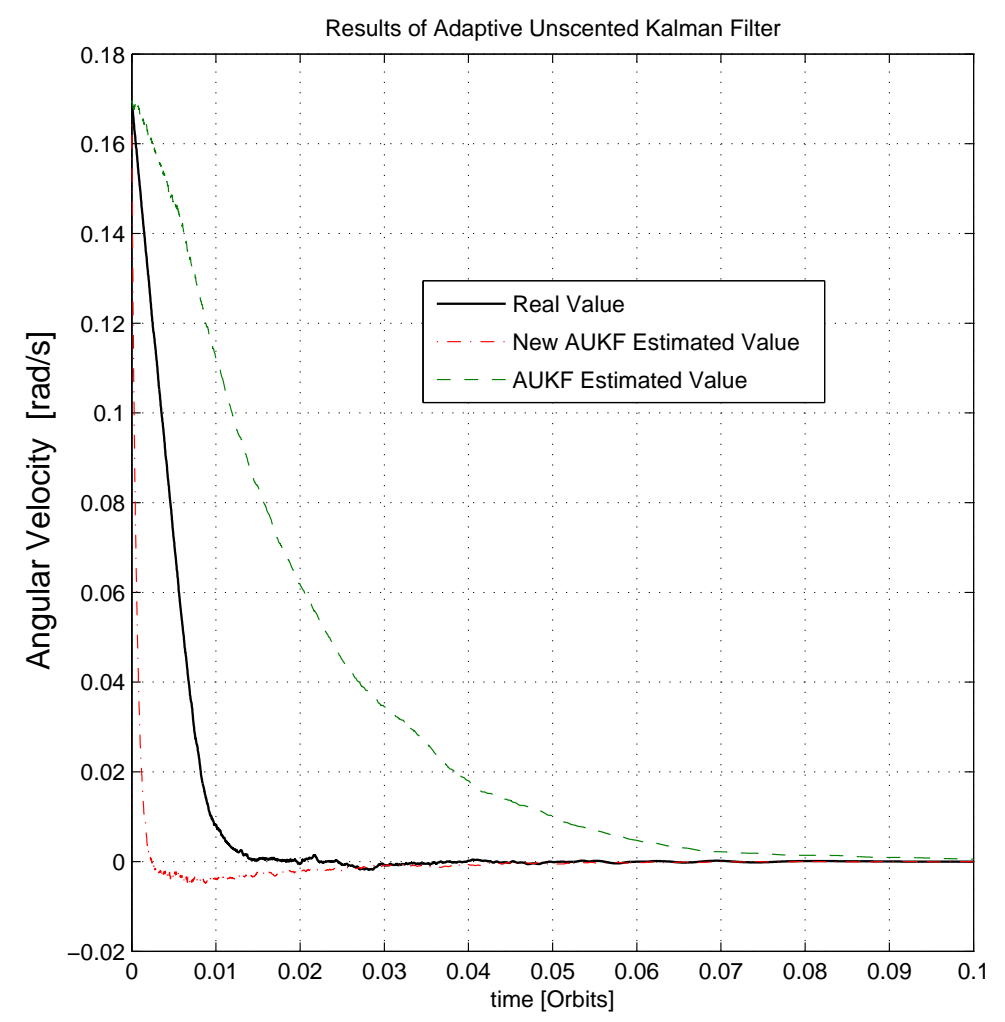

Figure 7. AUKF Estimated Angular Velocity of Satellite Z Axis

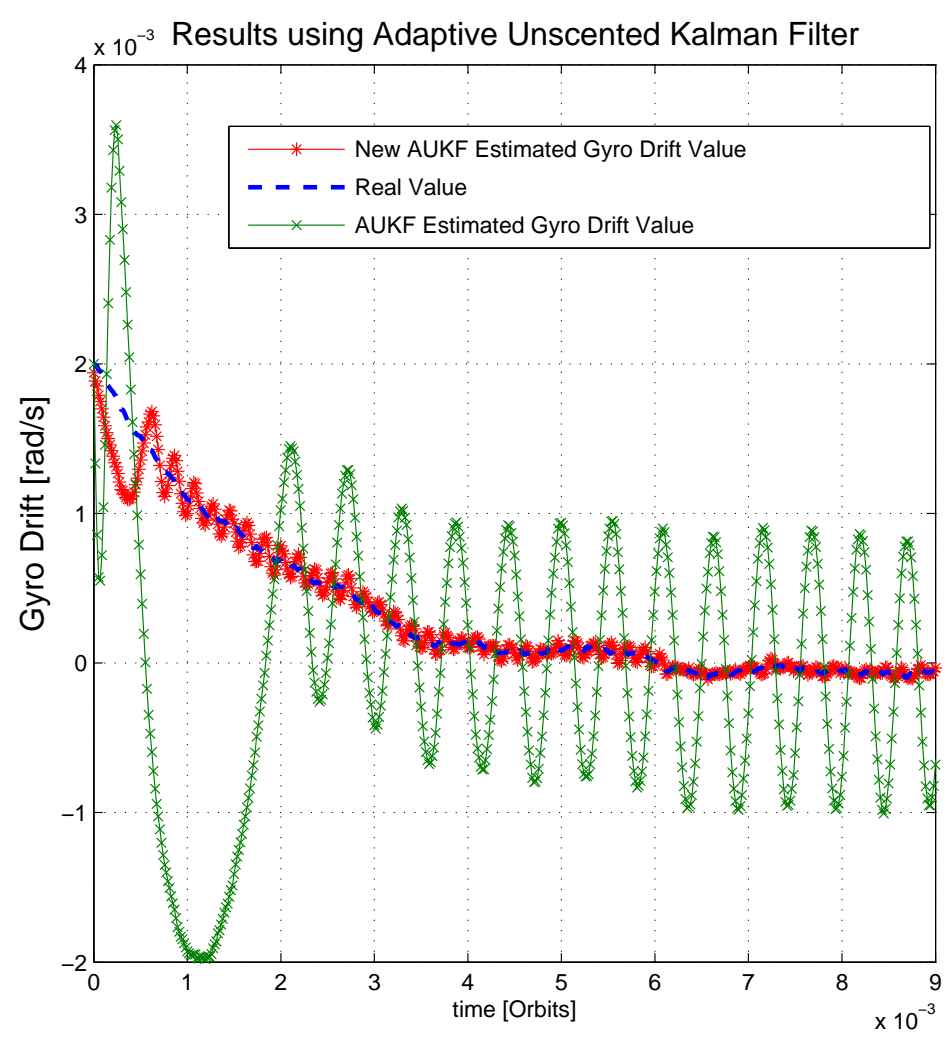

Figure 8. AUKF Estimated Gyro Drift of Satellite Z Axis 
$t=20-120$ s to better illustrate the differences between the controllers. Figure 10 shows the satellite angle for this test. The AFSMC controller provides smooth adjustment for the fault and demonstrates better control of the system overall without extensive tuning, as is required for the PID controller.

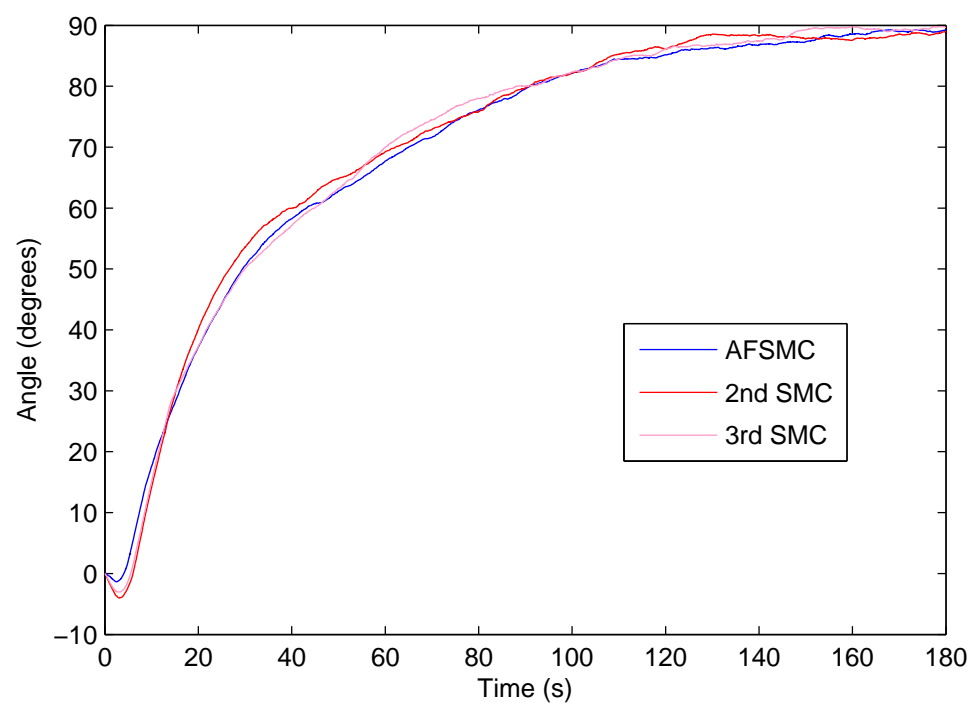

Figure 9. Nanosatellite 90 Degree Slew Angle with No Faults for AFSMC, 2nd SMC, and 3rd SMC Control

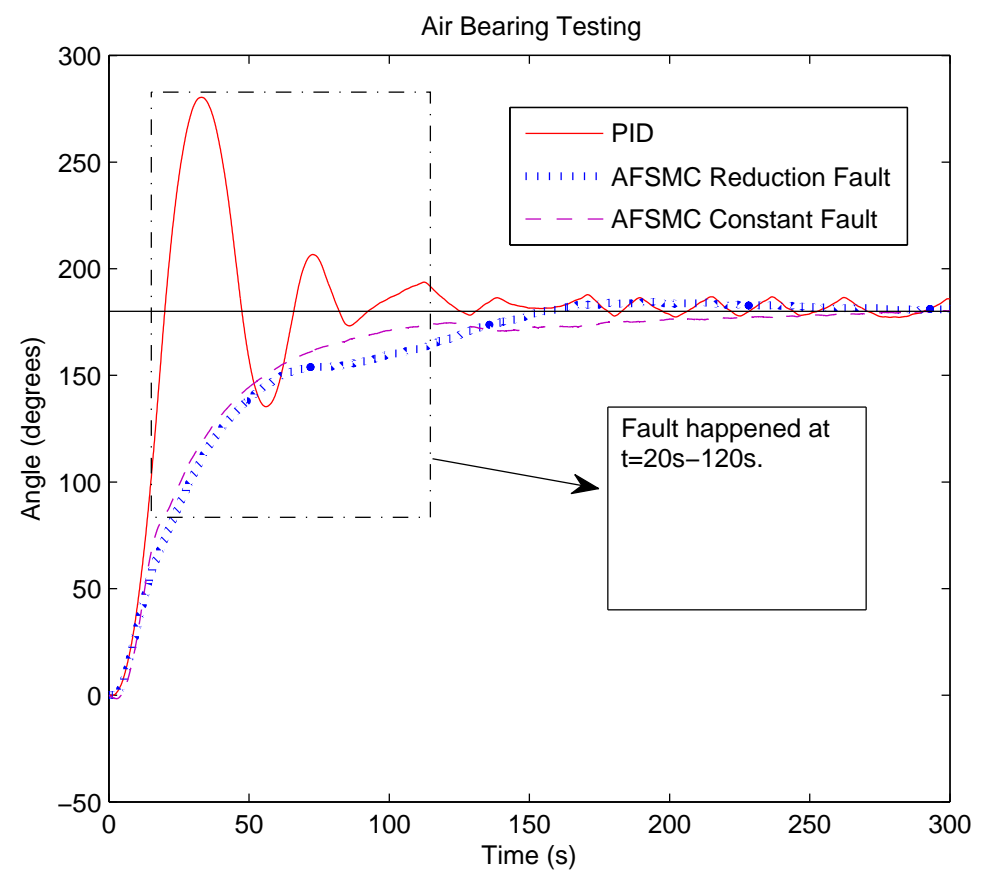

Figure 10. Nanosatellite 90 Degree Slew Angle with Wheel Fault for AFSMC and PID Control

After further controller tuning, three-axis control was tested using separate controllers and filters for each axis of rotation. The same 90 degree slew maneuver was performed, but with all axes under closed-loop control. Due to the difficulty of perfectly balancing the air bearing system in three axes simultaneously, a small disturbance torque can be considered to be present about the $\mathrm{X}$ and $\mathrm{Y}$ axes in these tests. Figure 11 shows the angular error in rotation about the $\mathrm{X}, \mathrm{Y}$, and $\mathrm{Z}$ axes for the PID controller (left) and the AFSMC controller (right) with no hardware faults present. Then, in Figure 12, a 25\% pitch wheel speed offset fault was added to the Y (pitch) axis between 20-120s. Similarly, in Figure 13, a $50 \%$ reduction in power was applied to the Z (yaw) axis between 20-120s. In all cases, the AFSMC controller achieves both higher accuracy in the presence of torque disturbances and faster compensation for actuator faults than the PID controller. 

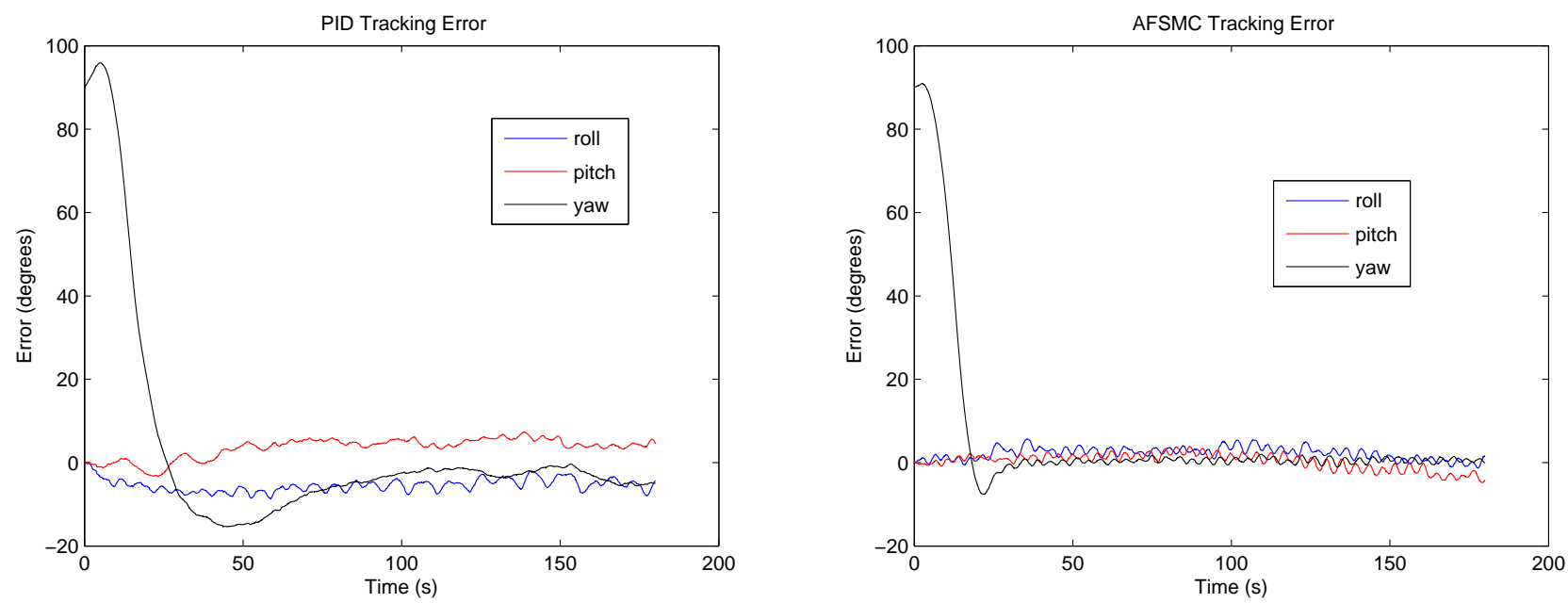

Figure 11. 3-axis Control 90 Degree Slew Maneuver with No Faults
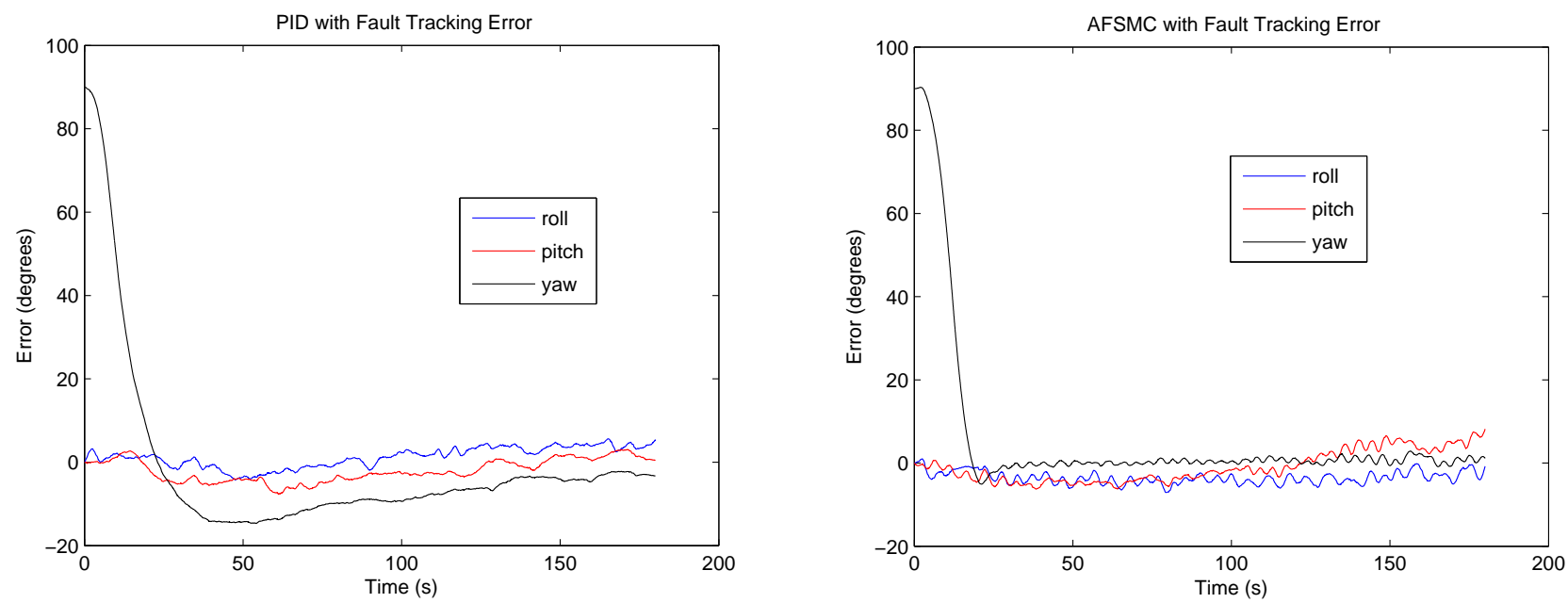

Figure 12. 3-axis Control 90 Degree Slew Maneuver with 25\% Pitch Wheel Offset Fault
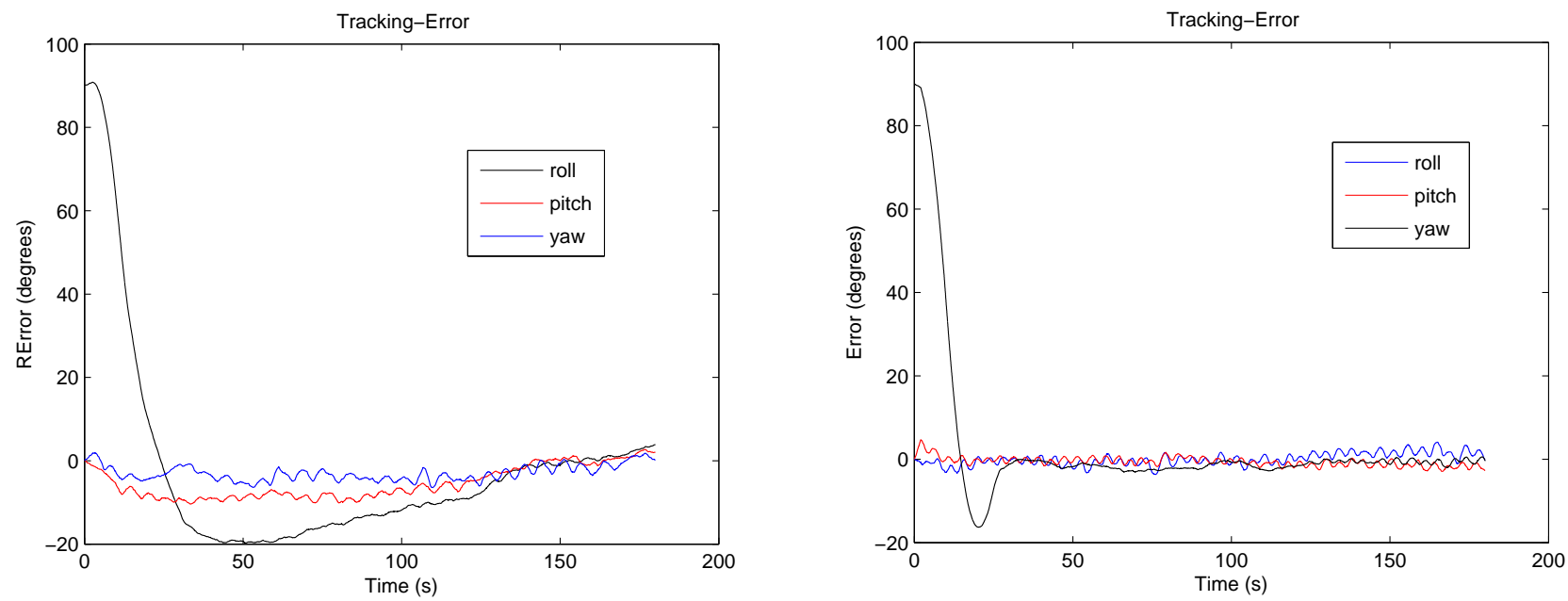

Figure 13. 3-axis Control 90 Degree Slew Maneuver with 50\% Yaw Wheel Power Reduction Fault 


\section{Conclusion}

In this research, an embedded implementation of nonlinear attitude estimation and control for nanosatellite hardware has been validated. A first-order adaptive fuzzy sliding mode control law was tested on an air bearing system, which uses an adaptive unscented Kalman filter with a new sigma point set to improve control performance. The proposed new adaptive Kalman filter was able to achieve greater estimation performance in simulation than the existing adaptive unscented Kalman filter. The complete embedded system was able to achieve accurate attitude tracking in both 1-axis and 3-axis attitude control tests on the air bearing hardware, and outperform a similar PID controller implementation under both normal and actuator fault conditions.

\section{References}

${ }^{1}$ Schwartz, J. L., Peck, M. A., and Hall, C. D., "Historical Review of Air Bearing Spacecraft Simulators," Journal of Guidance, Control and Dynamics, Vol. 26, No. 4, 2003, pp. 513-522.

${ }^{2}$ Li, J. Q., Post, M. A., and Lee, R., "Nanosatellite Attitude Air Bearing System using Variable Structure Control," IEEE 25th Annual Canadian Conference on Electrical and Computer Engineering, Montreal, Canada, April. 29- May. 2, 2012.

${ }^{3}$ Horri, N. M., Plamer, P. L., and Robers, M., "Energy Optimal Spacecraft Attitude Control Subject to Convergence rate Constraints," Control Engineering Practice, Vol. 19, 2011, pp. 1297-1314.

${ }^{4}$ Crassidis, J. L., Markley, F. L., and Cheng, Y., "Survey of Nonlinear Attitude Estimation Methods," Journal of Guidance, Control and Dynamics, Vol. 30, No. 1, 2007, pp. 12-28.

${ }^{5}$ VanDyke, M. C., Schwartz, J. L., and Hall, C. D., "Unscented Kalman Filtering for Spacecraft Attitude State and Parameter Estimation," Proceedings of AAS/AIAA Space Flight Mechanics Meeting, 2004, pp. 217-228.

${ }^{6}$ A. H. Mohamed, K. P. S., "Adaptive Kalman filtering for INS/GPS," Journal of Geodesy, Vol. 73, No. 4, May 1999, pp. 193-203.

${ }^{7}$ Soken, H. E. and Hajiyev, C., "Adaptive Unscented Kalman Filter with Multiple Fading Factors for Picosatellite Attitude Estimation," 4th International Conference on Recent Advances in Space Technologies, 2009, pp. 541-546.

${ }^{8}$ Abreu, N., "Fault Diagnosis With Adaptive Kalman Filters and CMG Design for Picosatellite ACS," MASC Thesis, Department of Aerospace Engineering, Ryerson University, 2011.

${ }^{9}$ Bae, J. H. and Kim, Y. D., "Attitude Estimation for Satellite Fault Tolerant System using Federated Unscented Kalman Filter," International Journal of Aeronautical and Space Science, Vol. 11, No. 2, 2010, pp. 80-86.

${ }^{10}$ Menegaz, H. M., Ishihara, J. Y., and Borges, G. A., "A New Smallest Sigma Set for the Unscented Transform and its Applications on SLAM," 2011 50th IEEE Conference on Decision and Control and European Control Conference, Orlando, Fl, USA, 2011, pp. 3172-3177.

${ }^{11}$ Xiong, K., Zhang, H. Y., and Chan, C. W., "Performance evaluation of UKF-based nonlinear filtering," Automatica, Vol. 42, 2006, pp. 261-270.

${ }^{12} \mathrm{Li}$, J. and Kumar, K. D., "Fault Tolerant Attitude Synchronization Control during Formation Flying," Journal of Aerospace Engineering, Vol. 24, 2011, pp. 251-263, doi: 10.1061/(ASCE)AS.1943-5525.0000080.

${ }^{13}$ Pukdeboon, C., Zinober, A. S. I., and Thein, M. W. L., "Quasi-Continuous Higher Order Sliding mode controllers for spacecraft attitude tracking maneuvers," IEEE Transactions on Industrial Electrionics, Vol. 57, No. 4, 2010 , pp. 1436-1444.

${ }^{14}$ Levant, A., "Quasi-continuous High Order Sliding Mode Controllers," IEEE Transactions on Automatic Control, Vol. 50, No. 11, 2005, pp. 1812-1816.

${ }^{15}$ Thienel, J. and Sanner, R. M., NASA techdocs, 2003.

${ }^{16}$ Ustrzycki, T., "Spherical Air Bearing Testbed for Nanosatellite Attitude Control Development," MASC Thesis, York University, 2011. 